\title{
Unraveling the role of nickel in the history of Earth and life
}

\author{
LAURA WASYLENKI', SHUI-JIONG WANG ${ }^{23}$ \\ School of Earth \& Sustainability and Dept. of Chemistry \& \\ Biochemistry, Northern Arizona University, Flagstaff, \\ Arizona 86011,USA, laura.w@nau.edu \\ ${ }^{2}$ China University of Geosciences, 100083 Beijing, China \\ ${ }^{3}$ GeoForschungsZentrum, 14473 Potsdam, Germany
}

Long-term evolution of Earth and its inhabitants could have unfolded in any number of different ways, but our planet's history followed the particular path that is now documented in the geologic record and that led to presentday conditions and biota. What factors controlled that evolution, shaping history as it actually occurred and ruling out other possible outcomes for the planet and for life?

Perturbations in the natural cycling of a particular metal, nickel, may well have steered the course of events at a couple of crucial junctures, because of nickel's essential role in certain enzymes, especially for methanogens and methane production. For example, a sharp decline in the marine concentration of $\mathrm{Ni}$ between 2.7-2.4 billion years ago likely contributed to a significant decrease in atmospheric methane production, enabling oxygenation of the planet and the onset of the first global ice ages [1,2]. About 252 million years ago, a sudden increase in marine $\mathrm{Ni}$ resulting from flood volcanism may have enabled proliferation of efficient, Ni-dependent metabolism of acetate by methanogens, thus driving global anoxia and the most severe of the Phanerozoic extinction events [3].

Unraveling how and when $\mathrm{Ni}$ and other trace metals were crucial factors in governing evolution will require resourceful interpretation and synthesis of many kinds of data. Our group leverages Ni stable isotope geochemistry to constrain how $\mathrm{Ni}$ cycling operates on the modern Earth surface and how it has changed throughout the history of Earth and life. Our approach combines analysis of natural samples and systematic laboratory experimentation aimed at elucidating reaction mechanisms relevant to $\mathrm{Ni}$ cycling. This talk will review highlights of recent efforts in this regard and some questions we aim to address in the near future.

[1] Konhauser et al., 2015, Astrobio; [2] Zahnle et al., 2006, Geobiology; [3] Rothman et al., 2014, PNAS. 